\title{
Editorial
}

\section{Learning and brain plasticity in mental disorders}

\author{
Herta Flor ${ }^{\mathrm{a}, *}$ and Andreas Meyer-Lindenberg ${ }^{\mathrm{b}, *}$ \\ ${ }^{a}$ Department of Cognitive and Clinical Neuroscience, Central Institute of Mental Health, Medical Faculty \\ Mannheim, Heidelberg University, Mannheim, Germany \\ ${ }^{\mathrm{b}}$ Department of Psychiatry and Psychotherapy, Central Institute of Mental Health, Medical Faculty Mannheim, \\ Heidelberg University, Mannheim, Germany
}

This special issue assembles studies that show the importance of learning and brain plasticity processes for the understanding of mental disorders. Most approaches to mental illness have examined the molecular or psychosocial factors underlying these disorders but have not focused on specific mechanisms such as learning or memory processes or brain plasticity nor have they examined these processes across disorders. Rather than taking a nosological approach, which leads to the problem of overlapping psychopathological characteristics and examining the neurobehavioral and molecular mechanisms of individual disorders, we suggest to determine similarities and differences of the underlying mechanisms of learning and neural plasticity across a variety of disorders. For example, rather than identifying genes related to anxiety disorder or addiction - very broad concepts - our approach focuses on genes related to Pavlovian fear conditioning and its extinction, glutamatergic signaling, plasticity of the amygdala or the orbitofrontal cortex, to

\footnotetext{
*Corresponding author: Herta Flor, PhD, Department of Cognitive and Clinical Neuroscience, Central Institute of Mental Health, Medical Faculty Mannheim, Square J5, D-68159 Mannheim, Germany. Tel.: +49621 1703 6302; Fax: +49621 1703 6305; E-mail: herta.flor@zi-mannheim.de; Andreas Meyer-Lindenberg, M.D, Ph.D, M.Sc, Department of Psychiatry and Psychotherapy, Central Institute of Mental Health, Medical Faculty Mannheim, Square J5, D-68159 Mannheim, Germany. Tel.: +49 06211703 2001; Fax: +49 6211703 2005; E-mail: a.meyer-lindenberg@zimannheim.de.
}

name just some examples of dysfunctional learning or plasticity mechanisms that could be examined. This approach is the basis of Collaborative Research Center 636 (www.sfb636.de) where we have mainly examined anxiety, depression, bipolar disorder, addiction and personality disorders.

The role of learning and memory mechanisms in mental disorder has been broadly discussed in the relevant literature of the past years, for example, with respect to anxiety disorders, addiction, depression or across disorders (e.g., Hsieh and Eisch, 2010; Lubin, 2011; Parsons and Ressler, 2013). In addition, a mechanistic approach to the analysis of genetic mechanisms has been advocated with a special emphasis on functional and structural circuit features of the human brain, as well as on gene-environment interactions (e.g., Meyer-Lindenberg and Weinberger, 2006). This combination of research approaches is at the forefront of recent approaches to mental disorder and meets the requirements of the NIMH Strategic Plan (National Institute of Mental Health, 2008). A specialty of this strategic plan is the development of new classifications of mental disorders based on behavioral and neurobiological mechanisms (e.g., Cuthbert and Insel, 2010; Cuthbert and Insel, 2013). The implementation of this goal has been termed the Research Domain Criteria Project and the NIMH now funds studies along these lines (http://www.nimh.nih.gov/researchfunding/rdoc.shtml). This new approach to mental 
disorder is in line with the mechanistic approach as outlined here - in fact, it could be argued that mechanisms must be the basis of a valid dimensional account of behavioral brain disorders.

This issue of Restorative Neurology and Neuroscience summarizes examples of a learning- and memory-based approach to mental disorder and its treatment from Collaborative Research Center 636 as well as other laboratories, with a focus on reports of the current research performed there. Section 1 covers mechanisms across several disorders and starts with a description of this approach by Morris, Rumsey and Cuthbert ("Rethinking mental disorders: the role of learning and brain plasticity"). Kuhn, Popovic and Pezawas ("Neuroplasticity and memory formation in major depressive disorder: an imaging genetics perspective on serotonin and BDNF") describe the important role of learning processes in their interaction with genetic risk variants on brain plasticity and symptom formation. In their contribution on bipolar disorder, Wessa, Kanske and Linke ("Bipolar disorder: a neural network perspective on a disorder of emotion and motivation") suggest that altered structural and functional connectivity, mainly between limbic and prefrontal brain areas, underlies emotional and motivational dysregulation in this disorder and might represent relevant vulnerability and disease markers. In an exemplary article ("Pathological anxiety and function/dysfunction in the brain's fear/defense circuitry") Lang, Bradley and McTeague show that startle modulation during imagery as an indicator of corticolimbic processing of fear-related memories is highly variable across anxiety disorders and greatly determined by comorbidity. Hamm, Richter, and Pané-Farré ("When the threat comes from inside the body: a neuroscience based learning perspective of the etiology of panic disorder") discuss how panic attacks can be conceptualized as an unconditioned circa defense response pattern to intense internal threat stimuli, characterized by strong autonomic surge and escape behavior and abnormal plastic changes of the brain. Panic disorder is then viewed as anxious apprehension after the experience of such severe panic attacks as conditioned responses to mild body symptoms, developing over time. Flor and Nees ("Learning, memory and brain plasticity in posttraumatic stress disorder: context matters") present data that show that enhanced cue and disturbed contextual conditioning and alterations in the hippocampus, amygdala and prefrontal cortex may be important determinants of the disorder, which are modulated by specific genetic variations and stress. Anderson and Kiehl ("Psychopathy: developmental perspectives and their implications for treatment") argue that psychopathy could best be treated when detected early since it is related to hypofunctioning of paralimbic circuits in the brain, which ordinarily support the integration of affective information into cognitive processes governing ongoing behavior and especially stimulus-punishment associations. For schizophrenia, Meyer-Lindenberg and Tost ("Neuroimaging and plasticity in schizophrenia") suggest a neurodevelopmental origin of the illness related to genetic and environmental risk factors that interfere with the structural and functional reorganization of neural networks at puberty. They propose an enduring disturbance of experience-dependent synaptic plasticity arising from developmental abnormalities in key neural circuits such as the dorsolateral prefrontal cortex and hippocampal formation, and their interactions. Genetic variants linked to neural plasticity modulate these circuits and non-invasive interventions such as transcranial magnetic stimulation can impact on the maladaptive changes. Finally, Farmer, Mansour, Baliki and Apkarian ("Chronic pain: the role of learning and brain plasticity") discuss how emotional learning defines the experience of chronic pain and how structural and functional changes related especially to limbic-prefrontal interactions shape chronicity.

In the second section of this issue we discuss the modulation of learning- and plasticity-related psychopathology, be it by biological or psychosocial factors or in the context of interventions. Due to the mechanism-based approach this strategy has the potential to accelerate the transfer of basic neuroscientific results into neurobehavioral or combined neurobehavioral and neuropharmacological treatment strategies. This mechanism-based approach is facilitated by the growing evidence that molecular and physiological mechanisms underlying neuronal plasticity and mechanisms of learning and memory share similar substrates. In the first article, Jensen and Walter ("Incentive motivational salience and the human brain") discuss how motivational changes and specifically the activity of the brain reward system and salience processing are related to psychopathology with a focus on psychosis and how these motivational dysregulations can be addressed with innovative psychological and pharmacological interventions. Ditzen and Heinrichs ("Psychobiology of social support: the social dimension of stress buffering") discuss how 
social support modulates the effects of stress on brain plasticity and demonstrate the relevance of these factors for the understanding of stress-related disorders as well as mental disorders that are related to social dysfunction. Paulzen and Gründer ("Effects of psychotropic drugs on brain plasticity in humans") suggest that many psychotropic drugs act upon synaptic plasticity and that new drugs that target plasticity should be developed. In an extension of these approaches, Hofmann, Fang and Gutner ("Cognitive enhancers for the treatment of anxiety disorders") describe how pharmacological agents that can enhance specific mechanisms of psychotherapy such as exposure have been developed that may lead to new advances in the treatment of anxiety disorders but also other mental disorders. In a similar direction, Poustka et al. ("Neurobiologically based interventions for autism spectrum disorders-rationale and new directions") suggest that a mechanism-oriented approach to autism spectrum disorder requires new interventions that alter the brain regions and interaction in the brain that underlie core mechanisms of the disorder and suggest neurofeedback as a viable strategy. Finally, Degen and Schröder ("Training-induced cerebral changes in the elderly") discuss maladaptive brain plasticity associated with normal and pathological aging and suggest innovative treatment approaches.

We hope that this special issue will provide an insight into mechanistic analyses of psychopathology that incorporate both behavioral and brain changes. The ultimate goal of this research effort is the exact description of the behavioral, neural and molecular deviations of learning and memory processes involved in the etiology of these disorders as well as the development of new assessment tools and mechanism-based behavioral and pharmacological treatment approaches.

\section{References}

Anderson, N.E. \& Kiehl, K.A. (2014). Psychopathy: Developmental perpectives and their implications for treatment. Restor Neurol Neurosci, 32(1), 107-113.

Cuthbert, B. \& Insel, T. (2010). The data of diagnosis: New approaches to psychiatric classification. Psychiatry, 73(4), 311314.

Cuthbert, B. \& Insel, T.R. (2013). Toward the future of psychiatric diagnosis: The seven pillars of RDoC. BMC Med, 11, 126.

Degen, C. \& Schröder, J. (2014). Training-induced cerebral changes in the elderly. Restor Neurol Neurosci, 32(1), 213-221.
Ditzen, B. \& Heinrichs, M. (2014). Psychobiology of social support: The social dimension of stress buffering. Restor Neurol Neurosci, 32(1), 149-162.

Flor, H. \& Nees, F. (2014). Learning, memory and brain plasticity in posttraumatic stress disorder: Context matters. Restor Neurol Neurosci, 32(1), 95-102.

Hamm, A.O., Richter, J. \& Pané-Farré, C.A. (2014). When the threat comes from inside the body: A neuroscience based learning perspective of the etiology of panic disorder. Restor Neurol Neurosci, 32(1), 79-93.

Hofmann, S.G., Fang, A. \& Gutner, C.A. (2014). Cognitive enhancers for the treatment of anxiety disorders. Restor Neurol Neurosci, 32(1), 183-195.

Hsieh, J. \& Eisch, A.J. (2010). Epigenetics, hippocampal neurogenesis, and neuropsychiatric disorders: Unraveling the genome to understand the mind. Neurobiol Dis, 39(1), 73-84.

Jensen, J. \& Walter, H. (2014). Incentive motivational salience and the human brain. Restor Neurol Neurosci, 32(1), 141-147.

Kuhn, M., Popovic, A. \& Pezawas, L. (2014). Neuroplasticity and memory formation in major depressive disorder: An imaging genetics perspective on serotonin and BDNF. Restor Neurol Neurosci, 32(1), 25-49.

Lang, P.J., McTeague, L.M. \& Bradley, M.M. (2014). Pathological anxiety and function/dysfunction in the brain's fear/defense circuity. Restor Neurol Neurosci, 32(1), 63-77.

Lubin, F.D. (2011). Epigenetic gene regulation in the adult mammalian brain: Multiple roles in memory formation. Neurobiol Learn Mem, 96(1), 68-78.

Mansour, A.R., Farmer, M.A., Baliki, M.N. \& Apkarian, A.V. (2014). Chronic pain: The role of learning and brain plasticity. Restor Neurol Neurosci, 32(1), 129-139.

Meyer-Lindenberg, A. \& Tost, H. (2014). Neuroimaging and plasticity in schizophrenia. Restor Neurol Neurosci, 32(1), 119-127.

Meyer-Lindenberg, A. \& Weinberger, D.R. (2006). Intermediate phenotypes and genetic mechanisms of psychiatric disorders. Nat Rev Neurosci, 7(10), 818-827.

Morris, S., Rumsey, J. \& Cuthbert, B. (2014). Rethinking mental disorders: The role of learning and brain plasticity. Restor Neurol Neurosci, 32(1), 5-23.

National Institute of Mental, Health. (2008). The National Institute of Mental Health Strategic Plan, Bethesda. MD: National Institute of Mental Health (NIH Publication 08-6368). http://www. nimh.nih.gov/about/strategic-planning-reports/index.shtml.

Parsons, R.G. \& Ressler, K.J. (2013). Implications of memory modulation for post-traumatic stress and fear disorders. Nat Neurosci, 16(2), 146-153.

Paulzen, M. \& Gründer, G. (2014). Effects of psychotropic drugs on brain plasticity in humans. Restor Neurol Neurosci, 32(1), 163-181.

Poustka, L., Brandeis, D., Hohmann, S., Holtmann, M., Bölte, S. \& Banaschewski, T. (2014). Neurobiologically based interventions for autism spectrum disorders-rationale and new directions. Restor Neurol Neurosci, 32(1), 197-212.

Wessa, M., Kanske, P. \& Linke, J. (2014). Bipolar disorder: A neural network perspective on a disorder of emotion and motivation. Restor Neurol Neurosci, 32(1), 51-62. 